Résumés des conférences et travaux

\title{
L'illustration des Livres Liturgiques français au Moyen Âge
}

\section{Alison Stones}

\section{OpenEdition}

\section{Journals}

Édition électronique

URL : https://journals.openedition.org/ashp/288

DOI : $10.4000 /$ ashp. 288

ISSN : 1969-6310

Éditeur

Publications de l'École Pratique des Hautes Études

Édition imprimée

Date de publication : 1 octobre 2008

Pagination : 175-180

ISSN : 0766-0677

Référence électronique

Alison Stones, "L'illustration des Livres Liturgiques français au Moyen Âge », Annuaire de l'École pratique des hautes études (EPHE), Section des sciences historiques et philologiques [En ligne], 139 | 2008, mis en ligne le 05 janvier 2009, consulté le 12 juillet 2021. URL : http://journals.openedition.org/ashp/ 288 ; DOI : https://doi.org/10.4000/ashp.288 


\title{
L’ILLUSTRATION DES LIVRES LITURGIQUES FRANÇAIS AU MOYEN ÂGE
}

\author{
Conférences de $\mathrm{M}^{\mathrm{me}}$ Alison Stones, \\ professeur à l'université de Pittsburgh, \\ directeur d'études invité
}

Les livres liturgiques illustrés ont joué un rôle central dans l'évolution de la peinture médiévale grâce à leur fonction à la fois pratique et symbolique dans la messe, l'office, et la célébration des autres sacrements de l'Église. Véhicules de la transmission des paroles divines et objets de culte en eux-mêmes, les livres liturgiques jouissaient d'une place favorisée dans la culture livresque chrétienne et les illustrations reflétaient la beauté des mystères de la foi. Certains manuscrits liturgiques avaient une double valeur emblématique, se rapportant non seulement à leur contenu mais aussi au rang et au pouvoir de leur commanditaire, étant surtout des livres de cérémonie, rarement utilisés, alors que d'autres, notamment les manuscrits de chant, servaient dans la liturgie de tous les jours comme en témoigne leur état de conservation, usés voire fragmentaires. Les livres liturques offrent aussi un fonds de modèles iconographiques et stylistiques qui servaient de base pour la création d'illustrations dans toutes les domaines du savoir médiéval, y compris les illustration de textes séculiers et en langue vernaculaires. Leurs usages liturgiques, contenant pour la plupart des éléments régionaux et locaux, en font des points de repère importants sur le plan géographique et chronologique par rapport aux autres types de livres illustrés apparentés.

Ces conférences ont présenté tout d'abord une esquisse des principales étapes de l'évolution chronologique de quatre genres de manuscrits liturgiques, à savoir les sacramentaires et les missels; les antiphonaires et les graduels; les bénédictionaux et les pontificaux; enfin les bréviaires et les martyrologes-obituaires, depuis les origines jusqu'à l'époque gothique. La seconde partie de chaque conférence a été consacrée à une étude détaillée du contexte artistique d'un choix de livres produits pour la plupart au temps des derniers Capétiens. Les manuscrits ont été présentés sous la forme de pages web (active server pages) créés en html en utilisant des images tirées de plusieurs bases de données, dont certaines sont disponibles en ligne comme Enluminures (IRHT-CNRS), Mandragore, Gallica et Banque d'images (BNF), British Library (Londres), Index of Christian Art (Princeton University), ainsi que des images numérisées d'après des livres ou d'après des photographies prises sur les originaux par l'auteur. Vu l'état précaire des obligations concernant les droits de reproduction dans la plupart des cas, les pages préparées pour ces séminaires ont pu être communiquées au cours des séminaires eux-mêmes et seront mises par la suite à la disposition des participants grâce au département d'histoire de l'art à l'université de Pittsburgh sur le site d'Alison Stones. 


\section{Les sacramentaires et les missels}

«Les sacramentaires et les missels manuscrits sont les sources principales de l'histoire de la messe et du missel. Si l'on veut suivre le développement liturgique du sacrifice chrétien, si l'on veut assister aux transformations du livre qui contient ses rites et ses formules, ses prières, c'est aux sacramentaires et aux missels manuscrits qu'il faut s'adresser. Ils sont les témoins qu'il convient d'interroger. Identifier ces documents, les dater, les analyer et les décrire constitue un travail de la plus haute importance; c'est le prélude obligé de toute recherche scientifique dans cet ordre des choses. »

C'est ainsi que commence l'introduction à l'ouvrage magistral qu'a consacré Victor Leroquais aux Sacramentaires et aux missels des bibliothèques publiques de France, paru en quatre tomes en 1924. C'était sa première grande étude consacrée aux livres liturgiques et dévotionnels français, où l'enluminure occupait déjà une place importante, le quatrième tome étant consacré entièrement à un choix de reproductions. Ses études consacrées aux psautiers, aux pontificaux, aux bréviaires des bibliothèques publiques de France et aux livres d'heures de la Biblliothèque nationale ont suivi jusqu'en 1942. Il convenait donc de commencer une série de séminaires sur les livres liturgiques par un examen des sacramentaires et des missels - tout en reconnaissant que beaucoup avait déjà été dit; les grandes lignes du développement, tant du point de vue du contenu que de celui de la décoration, avaient déjà été établis. On risquait de ne faire que répéter les jalons déjà énoncés par les spécialistes. Que restait-il à faire? De nos jours, nous disposons de maintes ressources qui n'étaient pas accessibles aux chercheurs travaillant à l'époque de Leroquais et de ses contemporains : les fac-similés d'une part et les banques d'images d'autre part, ces dernières de plus en plus accessibles par le moyen du web. Cela permet de considérer les missels - comme d'autres manuscrits enluminés d'une manière plus détaillée et plus comparative qu'auparavant. Il devient de plus en plus possible de situer les missels et les autres livres liturgiques dans un contexte culturel et artistique beaucoup plus large mais aussi plus précis.

Véhicules par excellence de la peinture en pleine page, à cause de représentation de la Crucifixion - présente dès les débuts du sacramentaire gélasien (VIII ${ }^{\mathrm{e}}$ siècle) sous forme symbolique et sous forme littérale à partir du sacramentaire de Gellone ( $\mathrm{VIII}^{\mathrm{e}}$ siècle) - miniature qui fait face par la suite au Christ en majesté, attesté dans l'iconographie pour la première fois au $\mathrm{IX}^{\mathrm{e}}$ siècle dans le sacramentaire de Metz, les sacramentaires et les missels permettent d'établir d'importants jalons dans l'évolution stylistique et iconographique de la peinture. C'est également au $\mathrm{IX}^{\mathrm{e}}$ siècle que le sacramentaire de Drogon, petit-fils de Charlemagne, introduit à la fois la typologie (les sacrifices de l'Ancien Testament sont représentés, par exemple, dans l'initiale historiée du Te igitur au canon de la messe). Les variantes dans le choix et le traitement de ces images, soit en pleine-page, soit sous forme d'initiales historiées, sont révélatrices d'un dynamisme souvent inattendu, alors que la qualité stylististique est souvent de très haut de gamme. À Cambrai au XII ${ }^{\mathrm{e}}$ siècle, le Christ en majesté cède la place à la Trinité-Trône de grâce (Cambrai, BM 234); à Corbie vers 1300 (Amiens, BM 157) c'est le couronnement de la Vierge qui est substitué au Christ en majesté, et cela dans un livre dont le décor peint souligne le rôle des saintes - Bathilde ( $\mathrm{VII}^{\mathrm{e}}$ siècle) est parmi celles dont la commémoration est dotée, exceptionnellement, d'une magnifique initiale historiée 
contenant le portrait de la sainte reine. Un splendide sacramentaire du $\mathrm{XI}^{\mathrm{e}}$ siècle, celui du Mont-Saint-Michel (Morgan Library M.641 / Rouen BM 116), contient une série de douze miniatures en pleine-page dont une représente Héraclius ramenant à Jerusalem la croix (dont le Mont-Saint-Michel possédait une relique) et une Assomption de la vierge très précoce; alors qu'à Saint-Denis c'est la communion miraculeuse du saint qui fait toute l'originalité du programme iconographique (BNF lat. 9436). Aux sacramentaires richement illustrés appartient le remarquable sacramentaire de la cathédrale de Limoges, aujourd'hui malheureusement endommagé, incommunicable et mal reproduit, où apparaît la lapidation du premier martyr, saint titulaire de la cathédrale de Limoges, à côté des principaux événements de la vie du Christ. Si, en général, les séries de miniatures font défaut dans les missels de l'époque gothique, les images traditionnelles de la Crucifixion et du Christ en Majesté prennent le relais, présentant une variété étonnante dans le détail, que ce soit par l'emploi de motifs particuliers, telle la Vierge qui tient - ou qui laisse tomber - un livre, ou l'épée qui lui perce le cœur selon la prophétie faite par Siméon lors de la Présentation au temple; le Christ en majesté trônant entre les tablettes de la loi et la calice et l'hostie; le Christ crucifié qui porte la couronne d'épines; Adam qui sort d'un tombeau aux pieds du Christ, parfois tenant un calice pour recueillir le sang du Seigneur, entre de nombeux autres points iconographiques divers. Le traitement géométrique ou naturaliste des scènes apporte une dimension stylistique de grande variété à l'époque gothique, où des missels très peu connus du Midi de la France offrent des points de repère à l'étude de l'enluminure dans toute la province ecclésiastique de Narbonne par exemple. C'est à l'époque gothique également que les images en pleine-page de la Crucifixion et du Christ en majesté sont réutilisées dans bien d'autres contextes manuscrits, comme pour témoigner de l'authenticité de leur contenu textuel, que ce soit un recueil des miracles de Notre-Dame par Gautier de Coinci (BNF, fr. 2163, copié en 1266), un recueil de textes dévotionnels (Lille, BM 607) ou bien les livres juratoires des consulats du Sud-Ouest (Agen, AD, ms. 42, Cahors, AM, ms. 1, Limoges, AC, AA 1, Perpignan, BM 70).

\section{Les graduels et les antiphonaires}

À l'époque gothique, les antiphonaires et les graduels sont dotés d'illustrations choisies en rapport avec les chants des grandes fêtes de l'année chrétienne et du culte des saints, contenant des initiales historiées à contenu narratif, à la différence des livres de chant des époques antérieures, où le décor, dans l'ensemble, se limite aux lettrines à motifs végétaux ou, plus sobrement encore, aux initiales filigranées dessinées à l'encre coloriée. Mais le problème de la survivance se pose en particulier pour les grands livres de chant qui, pour la plupart, ont terminé leur vie comme abat-jour ou livres composés de découpages et cela même sous les mains du célèbre historien de l'art John Ruskin, «C'était un dur boulot que de découper l'antiphonaire de Beaupré », a écrit Ruskin dans son journal! Les quelques parties qui survivent de ce magnifique antiphonaire, copié en 1290, et de celui de son prieuré dépendant Cambron, également transformé en scrapbook, sont des témoins importants quoique partiels. Ils soulèvent des questions concernant le rôle de l'enluminure chez les cisterciens en dépit des interdictions du $\mathrm{XII}^{\mathrm{e}}$ siècle, par rapport aux autres ordres. D'autres graduels cisterciens du 
$\mathrm{XIII}^{\mathrm{e}}$ siècle sont conservés à Laon, alors qu'on a un graduel fait pour les dominicaines de Metz conservé à Bruxelles, où apparaît l'iconographie du cœur de la Vierge transpercé par l'épée, motif qui se retrouve dans bien d'autres contextes liturgiques et qui a été favorisé par les ordres mendiants, soit dans leurs missels, soit à l'occasion dans une bible comme celle faite pour les dominicains de Metz conservée à Aschaffenburg. L'antiphonaire de Fontevrault, conservé à Limoges, retient l'attention grâce à sont état remarquable de conservation, à la densité de son illustration et à plusieurs choix iconographiques insolites, comme le portrait de sainte Radegonde, les nonnes devant la Vierge à l'enfant, un appui précoce à la fête de la Conception de la Vierge, ainsi que plusieurs images se rapportant à la bénédiction du cierge pascal.

À l'époque romane, par contre, ce sont les tropaires, prosaires et tonaires du SudOuest qui l'emportent par la remarquable qualité de leur décor peint, dont certains sont maintenant disponibles dans leur intégralité sous forme d'excellents facsimilés (le graduel de Gaillac, BNF, lat. 776, et le tropaire de Moissac, BNF Nouv. acq. lat 1871). C'est là que le magnifique décor végétal s'épanouit à défaut du narratif, pratique qui se rencontre également au XII ${ }^{\mathrm{e}}$ siècle dans le Codex Calixtinus toujours conservé dans les archives de la cathédrale de Santiago, qui réunit les chants de la messe et de l'office pour toutes les fêtes de saint Jacques de Compostelle, et qui fut confectionné sinon en France, du moins par un copiste et un enlumineur français. Toutefois le graduel de Saint-Denis (Bibl. Mazarine 384), contenant plusieurs grandes initiales historiées, témoigne dans le Nord au début du $\mathrm{XI}^{\mathrm{e}}$ siècle de l'émergence d'illustration narrative à l'époque romane; il a également fait l'objet d'un fac-similé récent. Mais d'autres manuscrits, conservés un peu partout dans les bibliothèques municipales de France, sont dans un état de conservation lamentable. Les antiphonaires et les graduels étaient des livres liturgiques essentiels, qui servaient vraiment. Car pour apprendre les chants, il fallait compter une dizaine d'années, nous dit Guido d'Arezzo.

\section{Les pontificaux et le rôle des évêques}

Faisant souvent partie d'un jeu de livres liturgiques commandé par des évêques particuliers, comme Nicolas de Fontaines et Enguerrand de Créquy, évêques de Cambrai, et leurs successeurs, Renaud de Bar, évêque de Metz, Guillaume de Thiéville, évêque de Coutances, les pontificaux contiennent des images se rapportant à la fois autant aux fêtes de l'année liturgique qu'aux fonctions de l'évêque. Il y a donc des rapprochements iconographiques et stylistiques à faire entre des scènes tirés de la vie du Christ entre pontificaux et évangéliaires, ainsi qu'avec les autres livres liturgiques, et pour les activités proprement épiscopales, entre différents pontificaux. Mais l'émergeance du pontifical proprement dit se révèle un phénomène tardif par rapport aux autres livres liturgiques, en comparaison avec l'Angleterre où les bénédictionnaires de Winchester et le pontifical de Sherborne témoignent d'une iconographie bien plus développée que dans les pendants français. Certes, le célèbre frontispice du sacramentaire de Marmoutiers conservé à Autun (Autun, BM 19 bis, avec d'excellentes reproductions sur Enluminures) témoigne dès le milieu du $\mathrm{IX}^{\mathrm{e}}$ siècle de tout l'intérêt qui fut porté aux fonctions des divers membres du clergé, alors que la place de l'alphabet en latin et en grec dans le décor du pontifical se manifeste dans le pontifical de Langres dès le $\mathrm{X}^{\mathrm{e}}$ siècle et fait de plus en plus l'objet d'une page décorée au XII ${ }^{\mathrm{e}}$ et début $\mathrm{XIII}^{\mathrm{e}}$ siècle (pontificaux de Chartres et de Reims). 
C'est dans le contexte de la représentation des fonctions de l'évêque que se distingue, au début du XIII ${ }^{\mathrm{e}}$ siècle, le pontifical à l'usage de Beauvais conservé à Besançon (excellentes reproductions sur Enluminures). Ce manuscrit mérite une étude approfondie, portant aussi bien sur ses rapports stylistiques avec le groupe des Bibles moralisées, les livres d'heures émergeants tels Morgan Library M. 92, les romans du LancelotGraal tels Rennes BM 255, texte où dans la partie « Estoire du saint Graal » il est question de la signification des vêtements de l'évêque et de ses fonctions ecclésiastiques tout comme dans le pontifical. Et c'est le pontifical de Beauvais qui, pour la première fois en France et bien avant l'Italie et le milieu papal, présente ces fonctions épiscopales dans un détail pictural extraordinaire au moyen d'une remarquable série d'initiales historiées, œuvre de deux peintres. On peut situer la production de ce manuscrit à Paris, juste après le quatrième concile du Latran tenu en 1215, dont les canons ont donné un élan à la production de manuscrits destinés aux laïcs et en même temps ont précisé encore plus les fonctions des membres de la hiérarchie ecclésiastique. C'est ainsi que d'autres pontificaux importants - celui de Châlons-sur-Marne, celui fait probablement pour Enguerrand de Créquy, évêque de Cambrai vers 1274, et conservé à Tolède, et celui fait au début du XIV ${ }^{\mathrm{e}}$ siècle pour Renaud de Bar, évêque de Metz, et maintenant divisé entre Cambridge et Prague - appuient, eux aussi, sur la représentation détaillée de toutes les fonctions de l'évêque. Les illustrations du pontifical de Châlons, sous forme de miniatures en pleine page, ont été remontées sur la reliure; pour le pontifical de Cambrai, on choisit les initiales historiées à antennes en forme de dragons ailés, réservant une miniature en pleine page pour l'alphabet tracé par terre en forme de sautoir par l'évêque même (et malheureusement très endommagée), alors que dans le pontifical de Renaud de Bar les enluminures prennent toute l'ampleur de miniatures rectangulaires accompagnés d'antennes souples, occupant la moitié des pages. Dans tous ces cas, comme pour le pontifical de Beauvais, le contexte artisque permet de suivre les peintres de ces manuscrits dans bien d'autres types de livres dont certains faits pour ces mêmes évêques.

IV. Des livres très communs et moins communs : les bréviaires, les psautiers, les rituels, processionnaux, martyrologes, obituaires

On peut dire que les psautiers les plus somptueux du Moyen Âge ont été faits pour des commanditaires ou des mécènes laïcs, surtout pour des femmes et que celles-ci, guidées peut-être par leurs confesseurs, ont pris une part active dans le choix des illustrations, car la variété en est extraordinaire. Il en est de même pour les psautierslivres d'heures et les livres d'heures sans psautier. En même temps, certaines régions, notamment aux environs de 1300, comme les diocèses de Thérouanne et d'Arras, ont vu produire d'énormes quantités de livres de dévotion illustrés, presqu'en série; il en était de même à Rouen, par exemple, à la fin du Moyen Âge. Le psautier, le psautierlivre d'heures et le livre d'heures ont pourtant occupé une place importante à côté des livres proprement liturgiques, n'ayant pas eux-mêmes servi directement dans la messe ni dans l'office. Ces livres de dévotions ont été le plus souvent dotées d'illustations importantes, dont des séries de miniatures en pleine page. Ces dermières manquent le plus souvent dans les bréviaires (à l'exception du frontispice du bréviaire de Philippe le Bel) qui, eux, sont normalement illustrés par des initiales historiées, sou- 
vent très endommagées car les bréviaires fait pour des clercs, comme c'était le cas des graduels et des antiphonaires, ont vraiment servi tous les jours. C'est sur les psautiers et les bréviaires qu'ont été calqués les livres d'heures, moyen de transmission de la liturgie propre au clergé jusqu'au cœur de la vie quotidienne des laïcs, surtout après la quatrième concile du Latran. Si les bréviaires se distinguaient rarement par leur enluminure avant le XIII ${ }^{\mathrm{e}}$ siècle - à l'exception du bréviaire de Montiéramey, BNF lat. 796 -, et si les commanditaires se limitaient normalement aux membres du clergé, le roi Philippe le Bel en fit un livre de prédilection pour les membres de la famille royale jusqu'à l'époque de Charles V.

D'autres catégories de livres liturgiques moins communs contiennent, à la différence des bréviaires, des enluminures aussi intéressantes qu'insolites, chacun étant un cas particulier. Ainsi le martyrologe-obituaire de Chartres, donné à la bibliothèque municipale de Chartres après la deuxième guerre mondiale, contient une série de dessins à la plume représentant les signes du zodiaque et les labeurs des mois - comptant parmi ces derniers plusieurs représentations uniques - les gémaux se cognant le front comme dans un geste de fraternité, un jeune homme assis tenant son cheval assoiffé par sa bride, l'achat de sangliers - sans parler du remarquable bifolio, éloge et portrait, ajouté au martyrologe après le décès de l'évêque Fulbert en 1027 (reproduit sur notre site internet http://images.library.pitt.edu/c/chartres). Au XIII ${ }^{\mathrm{e}}$ siècle, le martyrologe de l'abbaye Notre-Dames-des-Prés de Douai se distingue par la représentation dans les initiales historiées et dans les marges des saints et saintes de l'année, alors qu'au début du XIV ${ }^{\mathrm{e}}$ siècle l'obituaire de Notre-Dame de Paris contient des portraits funéraires de ses évêques (sur des feuillets ajoutés). Plusieurs bréviaires témoignent de la vitalité des régions dans la production livresque où les bréviaires illustrés font partie de toute une gamme de livres illustrés liturgiques aussi bien que non-liturgiques. Le bréviaire de Renaud de Bar, apparenté stylistiquement à son pontifical, son missel et son rituel détruit en témoignent à l'Est, alors que le bréviaire d'Agen, dont la partie conservé à Londres contient le nom du copiste Amigotus, permet de cerner tout un groupe de manuscrits provenant du Sud-Ouest et plus particulièrement de Toulouse.

\section{Conclusion}

Les livres liturgiques représentent un fonds incomparable pour l'étude de l'enluminure du Moyen Âge dans tous ses aspects et fournissent pour la plupart des repères fondamentaux pour la production livresque et les centres de production et de dissémination des livres et de l'enluminure. Ces genres de livres peuvent encore être exploités pour leur contribution à l'étude comparative de l'enluminure et de la peinture du Moyen Âge. À partir de la fin du XII ${ }^{\mathrm{e}}$ siècle et surtout dans les siècles suivants, les peintres de livres liturgiques ainsi que leurs modèles iconographiques et stylistiques vont se retrouver dans d'autres contextes, permettant ainsi de reconstruire l'œuvre de peintres et les commandes de mécènes. C'est dans cette direction que tendent de plus en plus les études pratiquées aujourd'hui grâce à la disponibilité de bases d'images fournissant de plus en plus de matière comparative peu connue ou inconnue. L'avenir des études fondées sur l'enluminure des manuscrits liturgiques est prometteur et s'étend bien au-delà de ces genres de livres proprement dits. 